\title{
Absorbed plant MIR2911 in honeysuckle decoction inhibits SARS-CoV-2 replication and accelerates the negative conversion of infected patients
}

\author{
Li-Kun Zhou ${ }^{1,2}$, Zhen Zhou', Xia-Ming Jiang ${ }^{3}$, Yishan Zheng ${ }^{4}$, Xi Chen', Zheng Fu', Gengfu Xiao ${ }^{3}$, Chen-Yu Zhang ${ }^{1}$, \\ Lei-Ke Zhang ${ }^{3}$ and Yongxiang $\mathrm{Yi}^{4}$
}

Dear Editor,

The Coronavirus disease 2019 (COVID-19) pandemic is one of the most serious global public health crises to date. As of July 12, 2020, more than 12.6 million cases of COVID-19 infection with 0.56 million deaths were confirmed worldwide ${ }^{1}$. Since there are no effective therapeutics to treat severe acute respiratory syndrome coronavirus 2 (SARS-CoV-2, the causative virus of COVID-19) infection so far, the pandemic is rapidly spreading worldwide. It is urgent to develop effective therapies, not only to treat infected patients but also to control the pandemic.

Our previous studies have demonstrated that a plant microRNA, MIR2911, which is enriched in honeysuckle decoction (HD), directly targets influenza A viruses (IAV), including $\mathrm{H} 1 \mathrm{~N} 1, \mathrm{H} 5 \mathrm{~N} 1$, and H7N9 subtypes by binding to their mRNA and blocking protein translation. Oral administration of HD can prevent IAV infection and reduce $\mathrm{H} 5 \mathrm{~N} 1$-induced mouse death ${ }^{2}$. Subsequent studies have shown that MIR2911 also directly inhibits the replication of various viruses in addition to $\mathrm{IAVs}^{3,4}$. Upon dietary uptake, these microRNAs self-assemble into

\footnotetext{
Correspondence: Chen-Yu Zhang (cyzhang@nju.edu.cn) or Lei-Ke Zhang (zhangleike@wh.iov.cn) or Yongxiang Yi (ian0216@126.com) ${ }^{1}$ Nanjing Drum Tower Hospital Center of Molecular Diagnostic and Therapy, State Key Laboratory of Pharmaceutical Biotechnology, Jiangsu Engineering Research Center for MicroRNA Biology and Biotechnology, NJU Advanced Institute of Life Sciences (NAILS), NJU Institute of Al Biomedicine and Biotechnology, School of Life Sciences, Nanjing University, Nanjing, Jiangsu 210023, China

${ }^{2}$ Tianjin Medical University Cancer Institute and Hospital, National Clinical Research Center for Cancer, Tianjin's Clinical Research Center for Cancer, Key Laboratory of Cancer Prevention and Therapy, Tianjin 300060, China Full list of author information is available at the end of the article These authors contributed equally: Li-Kun Zhou, Zhen Zhou, Xia-Ming Jiang, Yishan Zheng
}

exosomes and are then secreted into the circulation and delivered into target tissues or specific cells, including the liver, lung, spleen, pancreas, and $\mathrm{T}$ cells ${ }^{5-7}$. Given the unique GC-enriched nucleotide composition of MIR2911 (GGCCGGGGGACGGACUGGGA) and after we analyzed the genome sequence of SARS-CoV-2, it is most likely that the virus genome contains MIR2911-binding sites and that MIR2911 can inhibit SARS-CoV-2 replication directly. In the present study, we assessed the inhibitory effect of absorbed MIR2911 in HD on SARS-CoV-2 replication and conducted a clinical study to investigate the efficacy of HD in COVID-19 patients.

By using bioinformatics analysis, we predicted that there are 179 putative MIR2911-binding sites in the SARSCoV-2 genome. Twenty-eight binding sites (Supplementary Table S1) were confirmed by classic luciferase assay (Supplementary Fig. S1), which are distributed widely in the virus genome (Fig. 1a), indicating that MIR2911 may be able to inhibit the translation of almost all the proteins of SARS-CoV-2.

In order to assess the direct effect of absorbed MIR2911 on SARS-CoV-2 replication, cellular exosomes were collected from culture medium of HEK293T cells transfected with synthetic MIR2911 or control non-coding RNA (ncRNA), as similar method to previous report ${ }^{5}$ (Fig. 1b). Isolated cellular exosomes with/without MIR2911 were separately pre-incubated with $5 \times 10^{4}$ Vero E6 cells (ATCC1586) in $0.25 \mathrm{ml}$ cell medium for $8 \mathrm{~h}$. After changing the culture medium, the cells were infected with SARS-CoV-2 (nCoV-2019BetaCoV/Wuhan/WIV04/2019 ${ }^{8}$ ) at a multiplicity of infection (MOI) of 0.01 . Efficacies were evaluated by quantification of viral copy numbers in the cell supernatant via quantitative real-time RT-PCR (qRT-PCR) at $24 \mathrm{~h}$ post infection (p.i.) (Fig. 1b). As shown in Fig. 1c, 


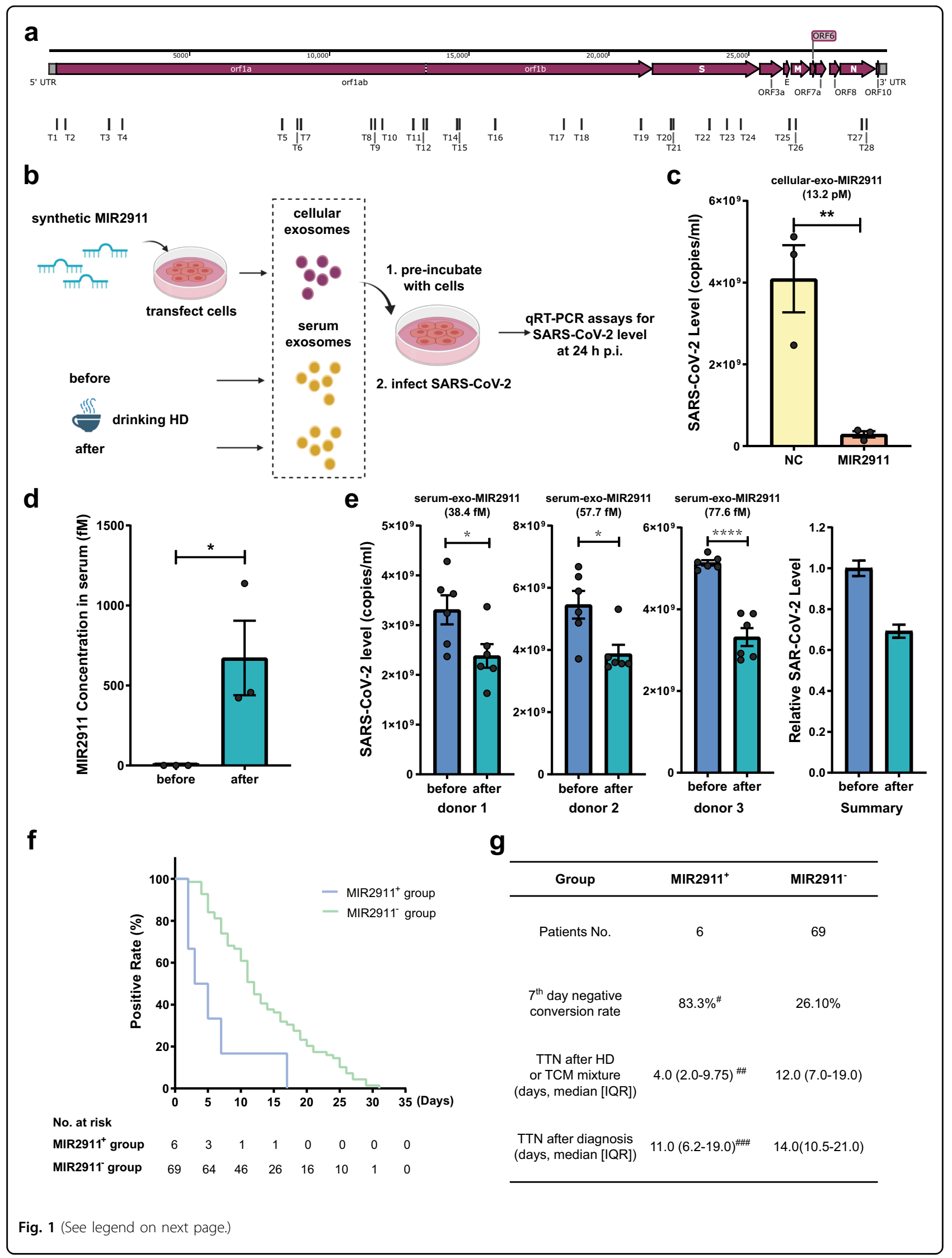


(see figure on previous page)

Fig. 1 Absorbed MIR2911 in HD directly inhibits SARS-CoV-2 replication. a Diagrams of MIR2911-binding sites on SARS-CoV-2 genome. b Diagrams of the collection of exosomes from cell medium or serum before or after the oral administration of HD and the measurement of the antiviral activities of cellular or serum exosomal miRNAs. c Efficacy of exosomes secreted by HEK293T cells transfected with MIR2911 or ncRNA in inhibiting replication of SARS-CoV-2. Vero E6 cells were pretreated with cellular exosomes for $8 \mathrm{~h}$, and virus was then added to incubate with cells at an $\mathrm{MOI}$ of 0.01 for $1 \mathrm{~h}$. Afterwards, exosomes and virus mixture was removed and the cells were cultured with fresh medium until the end of the experiment. Virus yield in the infected cell supernatants was quantified by qRT-PCR $(n=3)$ at $24 \mathrm{~h}$ p.i. d, e Efficiency of exosomal-MIR2911 in human serum to inhibit replication of SARS-CoV-2. Serum exosomes were harvest from three donors before and $2 \mathrm{~h}$ after oral administration of HD (10.5 pmol MIR2911/200 ml, once/30 g dry honeysuckle/per donor). MIR2911 concentration in serum were measured by qRT-PCR (d). Vero E6 cells were pretreated with human serum exosomes for $8 \mathrm{~h}$, and virus was then added to incubate with cells at an MOl of 0.01 for $1 \mathrm{~h}$. Afterwards, exosomes and virus mixture was removed and the cells were cultured with fresh medium until the end of the experiment. Virus yield in the infected cell supernatants was quantified by qRT-PCR $(\mathbf{e}, n=6)$ at $24 \mathrm{~h}$ p.i. $\mathbf{f}, \mathbf{g}$ Comparison of TTN $(\mathbf{f}, \mathbf{g})$ and 7 th day negative conversion rate $(\mathbf{g})$ between

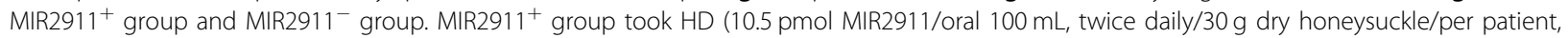

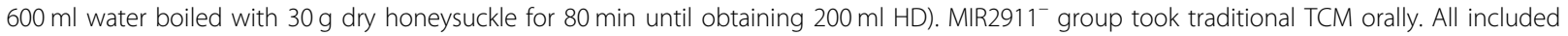

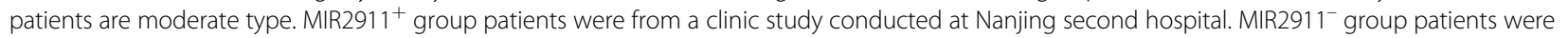
from the same hospital. Seventh day negative conversion rate defined as the percentage of patients converted to SARS-CoV-2 PCR-negative at the 7th day. The day patients received first dose of HD or TCM mixture was recorded as 1st day. Viral negative results must be confirmed twice of 24-h interval. The data are expressed as means \pm SEM. ${ }^{*} P<0.05,{ }^{* * *} P<0.0001$ using two-tailed Student's $t$ test; ${ }^{\#} P=0.004$ compared with MIR2911 ${ }^{-}$ group using $x^{2}$ test; ${ }^{\# \#} P=0.005$ compared with MIR2911- group (HR 0.28, 95\% Cl $0.12-0.67$ ) using Cox regression with the adjustment of sex factor; $\# \# P>0.05$ compared with MIR2911- group (HR 0.61, 95\% Cl 0.68-3.94) using Cox regression with the adjustment of sex factor. HD honeysuckle decoction, TTN time taken to become SARS-CoV-2 PCR-negative, TCM traditional Chinese medicine, IQR interquartile range, $\mathrm{HR}$ Hazard ratios, Cl confidence intervals.

cellular exosomal-MIR2911 at $13.2 \mathrm{pM}$ concentration inhibited $93 \%$ virus replication (from $4.09 \times 10^{9}$ to $2.87 \times$ $10^{8}$ copies/ml), indicating that absorbed MIR2911 directly and sufficiently inhibits SARS-CoV-2 replication (Fig. 1c). Next, we assessed the inhibitory effect of absorbed MIR2911 in HD on SARS-CoV-2 replication. Since absorbed MIR2911 in HD is delivered into the lung by exosomes through circulation, isolated exosomes from $62.5 \mu \mathrm{l}$ of donor serum before and after drinking $200 \mathrm{ml} \mathrm{HD}$ (30 g dry honeysuckle) separately were pre-incubated with $5 \times 10^{4}$ Vero E6 cells, as similar condition to that of cellular exosomal-MIR2911 (Fig. 1b). The MIR2911 concentration in $200 \mathrm{ml} \mathrm{HD}$ was $52.5 \mathrm{pM}(10.5 \mathrm{pmol} / 200 \mathrm{ml} / 30 \mathrm{~g}$ dried honeysuckle). Serum levels of MIR2911 in three heathy receivers after drinking for $2 \mathrm{~h}$ were $0.42,0.45$, and $1.13 \mathrm{pM}$ (Fig. 1d), which were undetectable before drinking). Exosomes (MIR2911 levels: before drinking, nondetectable; after drinking, they were 9.9, 14.4, and 19.4 amol) were preincubated with $5 \times 10^{4}$ cells (Fig. 1e, $\sim 180$ copies of MIR2911/cell). As shown in Fig. 1e, exosomes containing MIR2911 significantly inhibited virus replication (Fig. 1e). There is no difference of cell viabilities between exosomes with/without MIR2911 collected from the same donor before and after drinking HD (Supplementary Fig. S2). These results strongly suggest that MIR2911 is sufficiently absorbed by consumers drinking HD and that the absorbed MIR2911 significantly blocks SARS-CoV-2 replication.

To assess the antiviral effect of MIR2911 in HD on COVID-19 patients, we conducted a clinical study. Seventy-five moderate type COVID-19 patients who received routine antiviral therapy (RT) at Nanjing Second Hospital from January 2020 to March 2020 were enrolled in this study. Patients were divided into two groups based on additional treatment with MIR2911 in HD $\left(\mathrm{MIR} 2911^{+}\right)$or traditional Chinese medicine (TCM) mixture (MIR2911 $)$ in addition to RT. The primary endpoint was the negative conversion rate on the 7 th day from the first treatment. There were 6 and 69 patients in the MIR2911 ${ }^{+}$and MIR2911- groups, respectively (Supplementary Tables S2-S4). The negative conversion rate on the 7 th day in the MIR2911 ${ }^{+}$group was $83.3 \%$, which was dramatically improved compared with that of patients treated with MIR2911- TCM (26.1\%, P=0.004) (Fig. 1g, f). The time taken to become SARS-CoV-2 PCR-negative (TTN) also favored patients treated with HD-MIR2911 (median 4.0 vs. 12.0 days, HR $0.28,95 \%$ CI $0.12-0.67, P=$ 0.005 ) (Fig. $1 \mathrm{~g}, \mathrm{f}$ ). The median TTN of male patients in MIR2911 $^{+}(1$ case) and MIR2911- $(38$ cases $)$ are 5.0 days and 11.0 days (HR $0.003,95 \%$ CI $0.000018-0.52, P=$ $0.027)$, respectively. The median TTN of female patients in MIR2911 ${ }^{+}$(5 cases) and MIR2911 ${ }^{-}$(31 cases) groups are 3.0 days and 12.0 days (HR $0.15,95 \%$ CI $0.031-0.68$, $P=0.014)$, respectively.

HD has been used to treat viral infections for a thousand years in China. Previous studies have demonstrated that MIR2911 (0.06-0.18 pmol/day) in 1-3 ml HD significantly inhibits influenza virus replication in $20 \mathrm{~g}$ mouse $^{2}$. In addition to the Pharmacopoeia of the People's Republic of China ${ }^{9}$, we chose $30 \mathrm{~g}$ dried honeysuckle (MIR 2911 level: $10.5 \mathrm{pmol}$ ) per day for use. Our results demonstrate that $30 \mathrm{~g}$ dried honeysuckle is safe for use (Supplementary Table S4) and has sufficient antiviral function (Fig. 1d, e). On the other hand, the data that MIR2911 ( 60 fM) in exosomes significantly inhibits virus 
replication not only confirms the extra-high antiviral activity of MIR2911 (compared with that of remdesivir: $3.7 \mu \mathrm{M}$ and Chloroquine: $10 \mu \mathrm{M})^{10}$ but also provides a novel strategy that using serum exosomes collected from healthy donor offers the most similar condition in vivo to assess the efficacy of potential drugs in vitro.

In summary, our results suggest that absorbed plant MIR2911 in HD inhibits SARS-CoV-2 replication and accelerates the negative conversion of infected patients. HD treatment might greatly help cure infected patients and stop the COVID-19 pandemic.

\section{Acknowledgements}

We thank Wei Ye, Yun Chi, Cong Cheng, Lanlan Ma, Guangchang Dai, and Qian Shi, from the Second Hospital of Nanjing, for their assistance in the clinical study (Chinese Clinical Study Register number, ChiCTR2000029822). In addition, we thank Dr. Zhibin Hu (Nanjing Medical University) for his comments on the paper. This work was supported by grants from the Chinese Science and Technology Major Project of China (2015ZX09102023-003), National Basic Research Program of China (973 Program) (2014CB542300 and 2012CB517603), National Natural Science Foundation of China (81250044 81602697 and 31742075) and the Natural Science Foundation of Jiangsu Province (BE2016737), the Fundamental Research Funds for the Central Universities (020814380146) and Jiangsu Provincial Medical Youth Talent (QNRC2016056).

\section{Author details}

${ }^{1}$ Nanjing Drum Tower Hospital Center of Molecular Diagnostic and Therapy, State Key Laboratory of Pharmaceutical Biotechnology, Jiangsu Engineering Research Center for MicroRNA Biology and Biotechnology, NJU Advanced Institute of Life Sciences (NAILS), NJU Institute of Al Biomedicine and Biotechnology, School of Life Sciences, Nanjing University, Nanjing, Jiangsu 210023, China. ${ }^{2}$ Tianjin Medical University Cancer Institute and Hospital, National Clinical Research Center for Cancer, Tianjin's Clinical Research Center for Cancer, Key Laboratory of Cancer Prevention and Therapy, Tianjin 300060, China. ${ }^{3}$ State Key Laboratory of Virology, Wuhan Institute of Virology, Center for Biosafety Mega-Science, Chinese Academy of Sciences, Wuhan, Hubei 430071, China. ${ }^{4}$ Department of critical Care Medicine and Nanjing infectious Disease Center, the Second Hospital of Nanjing, Nanjing University of Chinese Medicine, Nanjing, Jiangsu 210003, China

\section{Author contributions}

Y.Y., L.-K.Zhang, and C.-Y.Z.conceived and designed the experiments; L.-K.Zhou, Z.Z., X.-M.J., Y.Z., and Z.F. participated in multiple experiments; L.-K.Zhou, Z.Z., G.
X., C.Z., L.-K.Zhang, and X.C. analyzed the data. L.-K.Zhou, Z.Z., C.-Y.Z., and L.-K. Zhang wrote the paper. C.-Y.Z., L.-K.Zhang, and Y.Y. provided the final approval of the paper.

\section{Conflict of interest}

The authors declare that they have no conflict of interest.

\section{Publisher's note}

Springer Nature remains neutral with regard to jurisdictional claims in published maps and institutional affiliations.

Supplementary Information accompanies the paper at (https://doi.org/ 10.1038/s41421-020-00197-3).

Received: 7 June 2020 Accepted: 12 July 2020

Published online: 05 August 2020

\section{References}

1. WHO. Coronavirus disease 2019 (COVID-19) situation report-174. https://www. who.int/docs/default-source/coronaviruse/situation-reports/20200712-covid19-sitrep-174 (2020)

2. Zhou, Z. et al. Honeysuckle-encoded atypical microRNA2911 directly targets influenza A viruses. Cell Res. 25, 39-49 (2015).

3. Huang, Y. et al. Honeysuckle-derived microRNA2911 directly inhibits varicella-zoster virus replication by targeting IE62 gene. J. Neurovirol. 25, 457-463 (2019).

4. Li, X. et al. Honeysuckle-encoded microRNA2911 inhibits Enterovirus 71 replication via targeting VP1 gene. Antivir. Res. 152, 117-123 (2018).

5. Zhang, L. et al. Exogenous plant MIR168a specifically targets mammalian LDLRAP1: evidence of cross-kingdom regulation by microRNA. Cell Res. 22, 107-126 (2012).

6. Zhao, C., Sun, X. \& Li, L. Biogenesis and function of extracellular miRNAs. ExRNA 1, 38 (2019).

7. Zhang, S. \& Hong, Z. Mobile RNAs — the magical elf traveling between plant and the associated organisms. ExRNA 1, 8 (2019).

8. Zhou, P. et al. A pneumonia outbreak associated with a new coronavirus of probable bat origin. Nature 579, 270-273 (2020).

9. Commission, C. P. Pharmacopoeia of the People's Republic of China, Vol. I 221 (People's Medical Publishing House, 2010).

10. Wang, M. et al. Remdesivir and chloroquine effectively inhibit the recently emerged novel coronavirus (2019-nCov) in vitro. Cell Res. 30, 269-271 (2020). 